\title{
Subthalamic Nucleus Local Field Potential Activity Helps Encode Motor Effort Rather Than Force in Parkinsonism
}

\author{
Huiling Tan, ${ }^{1,2}$ Alek Pogosyan, ${ }^{1,2}$ Keyoumars Ashkan, ${ }^{3}$ Binith Cheeran, ${ }^{2}$ James J. FitzGerald, ${ }^{2}$ Alexander L. Green, ${ }^{2}$ \\ Tipu Aziz, ${ }^{2}$ Thomas Foltynie, ${ }^{4}$ Patricia Limousin, ${ }^{4}$ Ludvic Zrinzo, ${ }^{4}$ and Peter Brown ${ }^{1,2}$ \\ ${ }^{1}$ Medical Research Council Brain Network Dynamics Unit at the University of Oxford, Oxford, OX1 3TH, ${ }^{2}$ Functional Neurosurgery, Experimental \\ Neurology Group, Nuffield Department of Clinical Neurosciences, John Radcliffe Hospital, University of Oxford, Oxford OX3 9DU, United Kingdom, \\ ${ }^{3}$ Department of Neurosurgery, Kings College Hospital, Kings College London, London SE5 9RS, United Kingdom, and ${ }^{4}$ Sobell Department of Motor \\ Neuroscience and Movement Disorders, University College London Institute of Neurology, London WC1N 3BG, United Kingdom
}

Local field potential (LFP) recordings from patients with deep brain stimulation electrodes in the basal ganglia have suggested that frequency-specific activities correlate with force or effort, but previous studies have not been able to disambiguate the two. Here, we dissociated effort from actual force generated by contrasting the force generation of different fingers while recording LFP activity from the subthalamic nucleus (STN) in patients with Parkinson's disease who had undergone functional surgery. Patients were studied while on their normal dopaminergic medication. We investigated the relationship between frequency-specific oscillatory activity in the STN and voluntary flexion of either the index or little finger at different effort levels. At each tested effort level (10\%, $25 \%$, and $40 \%$ of the maximal voluntary contraction force of each individual finger), the index finger generated larger force than the little finger. Movementrelated suppression of beta-band power in the STN LFP was significantly modulated by effort, but not by which finger was used, suggesting that the beta suppression in the STN LFP during sustained contraction serves as a proxy for effort. The absolute force scaled with beta power suppression, but with the scaling determined by the maximal voluntary contraction force of the motor effector. Our results argue against the hypothesis that the basal ganglia are directly involved in the parameterization of force during movement and support a role of the STN in the control of motor effort to be attributed to a response.

Key words: basal ganglia; beta oscillation; force generation; local field potentials; motor effort

\section{Introduction}

How do we scale our movements? The simplest policy would be to parameterize absolute force directly, but psychophysical arguments (Carson et al., 2002; Simon and Ferris, 2008) and some computational models (Guigon et al., 2007; Baraduc et al., 2013) suggest that we might scale movements by coding a more complex construct termed motor effort or sometimes vigor (Mazzoni et al., 2007). This can be considered to determine the gain of the motor command. Ordinarily, the dynamic range of motor effort will correspond to the range of strength of a given muscle group or effector, from no effort and no contraction to maximal effort and maximal voluntary contraction. Therefore, exerting a given force with a weaker digit such as the little finger requires more

Received Nov. 4, 2014; revised Feb. 17, 2015; accepted Feb. 22, 2015.

Author contributions: H.T., A.P., and P.B. designed research; H.T., A.P., K.A., B.C., J.J.F., A.L.G., T.A., T.F., P.L., L.Z., and P.B. performed research; H.T. and A.P. contributed unpublished reagents/analytic tools; H.T. analyzed data; H.T. and P.B. wrote the paper.

This work was supported by the Medical Research Council and performed in the National Institute for Health Research Cognitive Health Clinical Research Facility (0xford, UK).

The authors declare no competing financial interests.

This article is freely available online through the J Neurosci Author Open Choice option.

Correspondence should be addressed to Huiling Tan, Nuffield Department of Clinical Neurosciences, University of Oxford, Level 6, West Wing, John Raddliffe Hospital, Oxford OX3 9DU, United Kingdom. E-mail: Huiling.tan@ndcn.ox.ac.uk.

DOI:10.1523/JNEUROSCI.4609-14.2015

Copyright $\odot 2015$ the authors $\quad 0270-6474 / 15 / 355941-09 \$ 15.00 / 0$ effort than exerting the same force with a stronger digit such as the index finger. The actual force generated scales with effort, but the slope of this relationship will vary with changes in maximal voluntary contraction strength due to changes in effector, fatigue, disease, or injury. But is there any evidence for a central signal that behaves in such a way, varying with effort rather than effector or absolute force?

Here, we demonstrate such neural activity in the subthalamic nucleus (STN) of the basal ganglia. The basal ganglia have long been associated with the scaling of effort or force. Imaging studies in healthy humans have suggested that basal ganglia nuclei, in particular the internal portion of the globus pallidus and the subthalamic nucleus (STN), are involved in encoding gripping force amplitude and rate (Spraker et al., 2007; Prodoehl et al., 2009). Neuronal recordings in monkeys have also suggested that the basal ganglia play an important role in the control of the scaling of motor responses measured in terms of their amplitude or velocity (DeLong et al., 1984; Turner and Anderson, 1997). More recently, direct recordings from humans suggest that power suppression in the beta band and power increase in the gamma band of the local field potential (LFP) picked up from the STN may correlate with forces or efforts made over the lower and higher effort ranges, respectively (Tan et al., 2013b). Other investigators have also reported that STN LFP activity is modulated by movement amplitude and velocity, but mainly in the gamma frequency band (Anzak et al., 2012; Brücke et al., 2012; Joundi et 
Table 1. Clinical details of patients

\begin{tabular}{|c|c|c|c|c|c|c|c|c|}
\hline Case & Site & Age & Disease duration & UPDRS III On & UPDRS III Off & First symptom & Primary reason for DBS & Medication (daily dose in mg) \\
\hline 1 & 1 & 63 & 14 & 24 & 35 & Right hand tremor & Tremor & Levodopa 150, ropinirole 23 \\
\hline 2 & 1 & 50 & 9 & 23 & 58 & Right shoulder stiffness & On/off fluctuations and tremor & Levodopa 800 \\
\hline 3 & 1 & 50 & 4 & $\mathrm{~N} / \mathrm{A}$ & $\mathrm{N} / \mathrm{A}$ & Right arm tremor & Tremor & Rotigotine 16 , levodopa 300, entacapone 600 \\
\hline 4 & 2 & 71 & 16 & 13 & 32 & $\begin{array}{l}\text { Loss of dexterity and reduced arm } \\
\text { swing }\end{array}$ & Off period bradykinesia & $\begin{array}{l}\text { Levodopa } 600 \text {, amantadine 200, ropinirole 24, } \\
\text { rasagiline } 1\end{array}$ \\
\hline 5 & 2 & 48 & 7 & 13 & 58 & Dragging left leg & Tremor and gait difficulty & Levodopa 1300 \\
\hline 6 & 2 & 44 & 14 & 11 & 34 & Left arm bradykinesia & Motor fluctuations and dyskinesia & $\begin{array}{l}\text { Levodopa } 350 \text {, pramipexole } 1.05 \text {, amantadine } \\
\quad 300\end{array}$ \\
\hline 7 & 2 & 66 & 9 & 24 & 63 & Shoulder pain/stiffness & Motor fluctuations & Levodopa 650, pergolide 9 \\
\hline 8 & 3 & 62 & 2.5 & 8 & 18 & Left side tremor, rigidity & Left sided tremor, rigidity & Levodopa 800 \\
\hline 9 & 3 & 58 & 10 & 20 & 42 & Leg cramp & Tremor & $\begin{array}{l}\text { Trihexyphenidyl } 2 \text {, levodopa } 450 \text {, rasagline } 1 \text {, } \\
\text { amantadine } 100\end{array}$ \\
\hline 10 & 3 & 62 & 10 & 8 & 20 & Left side tremor and bradykinesia & $\begin{array}{l}\text { On off fluctuations, tremor and impulse } \\
\text { control disorder }\end{array}$ & Levodopa 1000, trihexyphenidyl 6 \\
\hline 11 & 3 & 49 & 10 & 6 & 42 & Tremor in right hand & Tremor & Levodopa 300, trihexyphenidyl 2 \\
\hline
\end{tabular}

Surgical sites: (1) Kings College Hospital, London; (2) National Hospital for Neurology and Neurosurgery, London, United Kingdom; (3) John Radcliffe Hospital, $0 x f o r d$. Cases 8 and 11 underwent unilateral implantation. Cases 10 and 11 were excluded from the final analysis because they made more than $20 \%$ invalid trials per session. N/A, Not available.

al., 2012), perhaps because the tasks tested required higher effort levels over which gamma-band changes may be more evident in the STN LFP. Moreover, in the paradigms studied thus far, the absolute force and other biomechanical parameters have not been disambiguated from motor effort. Here, we explore whether task-related beta-band desynchronization in the STN LFP scales with motor effort during low-intensity contractions and if this can be disambiguated from a scaling with force per se through the selection of different motor effectors. Our findings have significant implications for the understanding of the normal functioning of the basal ganglia and for theoretical accounts of motor impairment in disorders such as Parkinson's disease (PD).

\section{Materials and Methods}

Subjects. Eleven patients with idiopathic PD (mean disease duration of 10 years, mean age 56.6 years, range $44-71$ years, eight males) provided written informed consent to take part in this study, which was approved by the local ethics committees. Nine patients underwent bilateral and two patients underwent unilateral implantation deep-brain stimulation (DBS) electrodes into the STN as a prelude to therapeutic high-frequency stimulation for advanced idiopathic PD with motor fluctuations and/or dyskinesia. These offered access to 20 STNs in total. Techniques to target and implant electrodes in the STN have been described previously (Foltynie and Hariz, 2010). Microelectrode recordings were not made during surgery. The permanent quadripolar macroelectrode used was Model 3389 from Medtronic Neurologic Division featuring four platinumiridium cylindrical surfaces. Its contacts are numbered $0,1,2$, and 3 , with 0 being the most caudal and 3 the most cranial. Localization was supported by the effects of direct stimulation (sites 1 and 3) intraoperatively and by immediate postoperative stereotactic imaging (site 2). DBS electrode extension cables were externalized through the scalp to enable recordings before connection to a subcutaneous DBS pacemaker implanted in a second operative procedure up to $7 \mathrm{~d}$ later. Clinical details of the patients are given in Table 1. The patients showed $61.2 \pm 4.4 \%(p<$ 0.001) improvement in the motor section of the Unified Parkinson's Disease Rating Scale (UPDRS) on treatment with levodopa, indicating good responsiveness to this drug.

Experimental paradigm. LFPs were recorded while patients engaged in a task in which they were requested to press a force-sensitive device

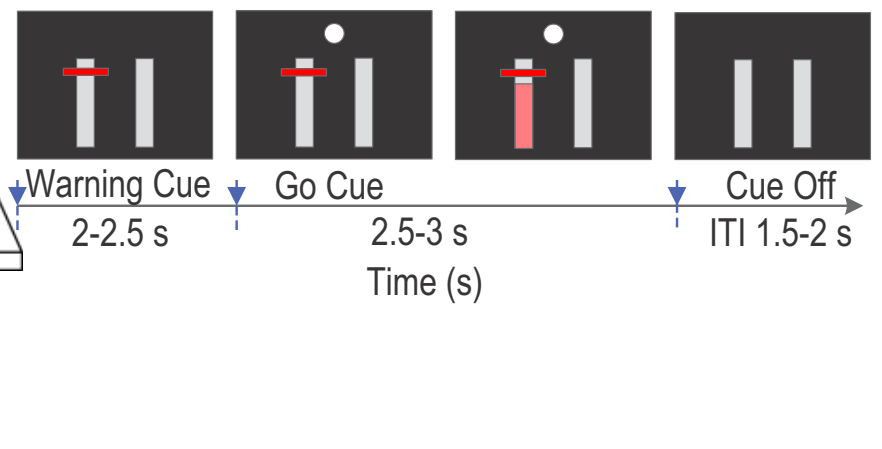

$2.5-3 \mathrm{~s}$ ITI 1.5-2 s

digm. Two vertical bars were presented, with the left and right denoting the little and index finger of the left hand or the index and little finger of the right hand. Filled pink color indicated the pressing force of the responding finger, cations (corresponding to $10 \%, 25 \%$, and $40 \%$ of MVC) on one of the vertical bars, indicating which finger to press and the effort level required. Subjects were asked to press with the correct finger with the appropriate level of effort at the onset of the filled circle go cue and to hold the force until the circle was extinguished.

(Precision Pinchmeter P200; Biometrics) with different fingers at different effort levels after imperative visual cues. Subjects were seated in a comfortable chair in front of computer monitor with their shoulders adducted and their elbows flexed at $\sim 90^{\circ}$. Subjects were asked to place their pronated hand flat on top of a desk with the flexor surface of the forearm and wrist touching the desk. All fingers were kept naturally extended and with a force-sensitive device under the index finger and little fingers. The experiment started with separately measuring the maximal voluntary contraction (MVC) pressing force of the index and little finger. The subjects were asked to press the force-sensitive device using the corresponding finger as hard as they could while keeping the position of the hand, wrist, and forearm relatively stable and touching the desk. Three attempts were recorded and the average of the maximal force achieved was used as the MVC for that finger. In the formal experiment, subjects were presented with a series of visual cues displayed on the monitor indicating which finger to press and the effort level that should be applied. These visual cues included two vertical bars that corresponded to the pressing force of the index and little fingers according to the position of the bars: for the right hand, the bar on the left side corresponded to the index finger and the bar on the right corresponded to the little finger. The converse was true for the left hand (Fig. 1). The pressing force of each finger was measured and displayed in real time as a filled pink color of the corresponding bar, with $50 \%$ of the MVC of that finger displayed as a completely pink bar. At the beginning of each individual trial, a horizontal line appeared on one of the vertical bars at one of the three vertical locations corresponding to $10 \%, 25 \%$, and $40 \%$ of MVC, 
indicating which finger should be pressed and the effort level to apply. Subjects were asked to prepare to press with the correct finger at the appropriate effort level once they saw the horizontal line, but only start to press once a circular white imperative signal appeared. The filled pink color in the vertical bar indicated whether pressing force reached the required level and subjects were asked to continue pressing until the imperative signal went off (Fig. 1). There were 100 trials in each session and the warning cues randomly changed in the six experimental conditions with $\sim 16$ trials in each condition: little finger at $10 \%$ of MVC, little finger at $25 \%$ of MVC, little finger at $40 \%$ of MVC, index finger at $10 \%$ of MVC, index finger at $25 \%$ of MVC, and index finger at $40 \%$ of MVC.

Recordings. Recordings were made when the patients were on their usual dopaminergic medication 3-6 d postoperatively while electrodes were externalized and before implantation of the pulse generator. Both hands were tested for patients who underwent bilateral implantation, with the order in which the left and right hands were tested counterbalanced across subjects. For patients who underwent unilateral implantation, only the hand contralateral to the side of electrode implantation was tested. Monopolar LFPs were recorded with a TMSi porti (TMS International) and its respective software. They were high- and low-pass filtered at 0.5 and $300 \mathrm{~Hz}$, respectively. Force was low-pass filtered at $200 \mathrm{~Hz}$. LFPs and force were sampled at $2048 \mathrm{~Hz}$.

Analysis. Analyses of both behavioral and LFP data were performed in MATLAB (version 2012b; The MathWorks). The finger-pressing force trajectory of each individual trial of each hand was normalized against the average MVC force of the index finger of that hand for easier comparison across subjects. The subjects were asked not to press until the visual "Go" white circle cue appeared so as to have a "preparation" phase between the warning cue and the go cue. Trials with muscle activation in this phase, measured as a pressing force $>3$ times the SD of the baseline, were rejected. Sessions with $>20 \%$ invalid trials were excluded and this afforded 16 hands from nine subjects for the final analysis. The majority of invalid trials were due to premature responses in the preparation phase. The mean number ( \pm SEM) of remaining trials per hand per condition was $14.0 \pm 0.5$. The force trajectories were differentiated against time and then low-pass filtered at $40 \mathrm{~Hz}$ to calculate the rate of change in force. Force initiation was defined as the time when the rate of change in force crossed a threshold of three times the SD of the signal (and its noise) at "rest" and was sustained above this threshold for at least $100 \mathrm{~ms}$. Reaction time was calculated as the interval between the Go cue and force initiation; the time to peak force rate was calculated as the interval between the force initiation and the time when the rate of change in force reached its maximum. Stable force was calculated as the average force during a second in each trial when the force was stable. All of these parameters were calculated for each individual trial and averaged within each experimental condition before averaging across subjects.

LFP data were converted offline to give three bipolar contact pairs ( 01 , 12 , and 23) per electrode. A time-frequency decomposition based on the continuous wavelet transform was then applied to the LFP recordings from each trial to analyze changes in LFP activity in the time-frequency domain. Event related LFP power change (ERPC) was subsequently normalized relative to the average power during the one second before the warning cue so that a value higher than zero indicated power higher than before the cue and vice versa. The normalized power of each bipolar contact pairs was aligned to either the warning cue (to calculate ERPC in the preparation phase) or the Go cue (to calculate ERPC in the pressing phase) and subsequently averaged across the three bipolar contacts for each STN lead contralateral to the gripping hand. Analysis of the LFP from the STN focused on the side of the STN that was contralateral to the hand used to perform the task. Of the three bipolar channels from each STN, the channel with the largest event-related power change in the beta-band $(13-30 \mathrm{~Hz})$ - that is, the largest difference between the trough of event-related desynchronization during the sustained contraction phase and the peak synchronization during a second after movement in the beta band (Devos et al., 2006) — was selected for further analysis. Postoperative MRI scans were available for coregistration with preoperative MRI in 12 of 16 STNs included in the final analysis. Within this group, the bipolar channels that showed the largest movement-related modulation in the beta-band either had one contact inside STN ( 7 of 12, with the other contact either above or lateral to STN) or had both contacts inside STN ( 5 of 12). In two of the STNs without postoperative MRI, the bipolar channels chosen for final analysis included one of the contacts used for chronic clinical stimulation. In a further patient (1 STN) without postoperative imaging, follow-up chronic stimulation details were unavailable. In the remaining STN, the lower most bipolar contact pair was used for analysis and the electrode subsequently repositioned by advancing it $5 \mathrm{~mm}$ deeper before connection to the subcutaneous pulse generator.

Statistics. Repeated-measure ANOVAs were used to examine the effect of effort level and the pressing finger on the force and LFP measurements. Correlation and partial correlation were used to investigate the relationship between normalized force and LFP measurements across subjects. These statistical tests were performed in SPSS Statistics 19. Independent software (G* power 3.1.9.2; Erdfelder et al., 1996; Faul et al., 2007) was used to calculate statistical power. Means \pm SEM are presented throughout the text unless otherwise specified.

A multilevel multivariate regression modeling approach (Hox, 2002) was used to investigate whether the frequency-specific STN LFP activity was related to required effort, actual effort, or exerted force and to identify the relationship between frequency-specific LFP activities and absolute exerted force within subjects and how this relationship changed with the motor effector (index or little finger). The multilevel multivariate regression modeling approach used here has the advantage of taking into account the dependency in the data caused by repeated measurements within subjects, which makes it possible to investigate within-subject correlations that are consistent across subjects. Multilevel modeling was performed using the nlme package (Pinheiro et al., 2013) in R (R Development Core Team, version 2.13.2). In the modeling, the absolute pressing force was the dependent variable, LFP measurements were the continuous independent variables, and the motor effector (index finger or little finger) was treated as a categorical independent variable. The effects of all of the predictors were treated as fixed effects (consistent across subjects) and the baseline difference between individual hands was treated as a random effect. The normality assumption of the modeling was assessed by visually inspecting the Q-Q plot of the residuals of the modeling; the residues were also plotted against the fitted values and a uniform distribution was used to assess the validity of the modeling. The fit of different models was compared using the likelihood-ratio test. For variables with a significant effect, the estimated regression coefficient (mean \pm SEM) and the $p$-value are reported.

Akaike information criterion (AIC) analysis was used to compare the different models used to describe the relationship between frequencyspecific LFP activities and effort and absolute force. The AIC value was first calculated for each model and the best candidate model with minimal AIC was identified. The differences in AIC with respect to the AIC of the best candidate model $\left(\Delta_{i}(A I C)=A I C_{i}-\min (A I C)\right)$ and the Akaike weight for a model $\left(w_{i}(\right.$ AIC $\left.)=\frac{\exp \left\{-\frac{1}{2} \Delta_{i}(A I C)\right\}}{\sum_{k=1}^{k} \exp \left\{-\frac{1}{2} \Delta_{k}(A I C)\right\}}\right)$ were then calculated and used to evaluate the probability that the $i$ th model was the best model in the AIC sense given the data and the set of candidate models (Burnham and Anderson, 2002; Wagenmakers and Farrell, 2004). The likelihood of the other models relative to the best candidate model was also evaluated using the relative Akaike weight $\frac{w_{i}(A I C)}{w_{b s t}(A I C)}$, where $w_{b s t}(A I C)$ was the Akaike weight of the best candidate model.

\section{Results}

\section{Index finger generates larger force compared with little finger} for a similar effort level

The mean MVC of the index finger $(30.4 \pm 3.14 \mathrm{~N})$ was significantly larger than the mean MVC of the little finger $(20.6 \pm 1.87$ $\mathrm{N}, t_{15}=4.255, p=0.001$ ), with the index finger $53.7 \pm 14.6 \%$ stronger than the little finger. The average reaction times for different experimental conditions are shown in Figure 2A. Twoway ANOVA with the effect of factors of finger (index or little) 
A

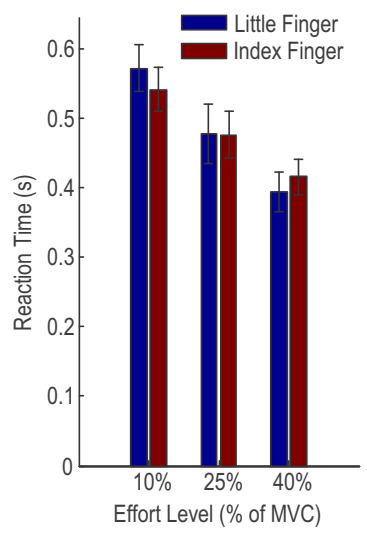

B

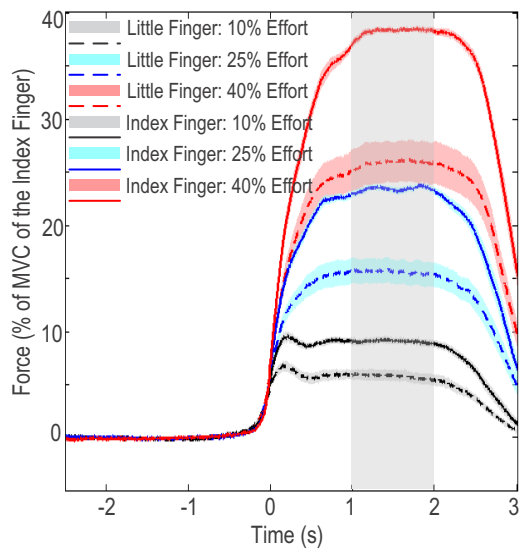

C

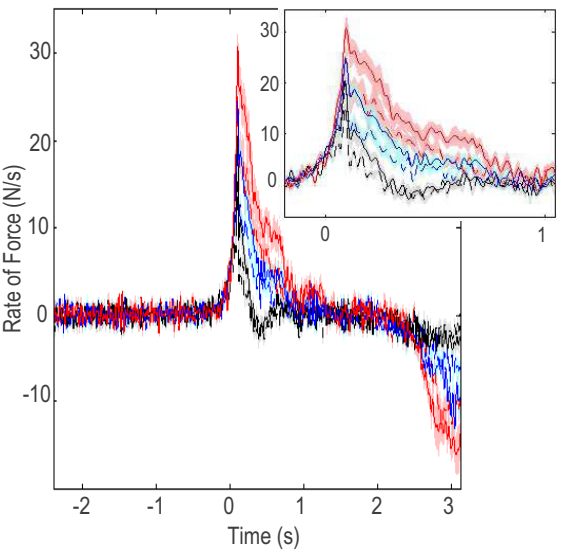

Figure 2. Behavioral results. $\boldsymbol{A}$, Reaction time reduced with increasing effort level, but was independent from motor effector. $\boldsymbol{B}$, Exerted force aligned to force onsets in different experimental conditions. The actual exerted force was modulated by the effort level required. At each effort level tested, the force achieved by the index finger was significantly larger than that achieved by the little finger, confirming successful dissociation between effort and actual force in this paradigm. $C$, The rate of force development aligned to force onsets in different experimental conditions. The inset shows that the time to peak force rate was constant across all experimental conditions, but the peak force rate changed with effort and motor effector. Group mean \pm SEM (error bars in $A$ and colored shaded areas in $\boldsymbol{B}$ and $\boldsymbol{C}$ are shown. Gray band in $\boldsymbol{B}$ is the period taken as stable force.

Little Finger: $10 \%$ Effort

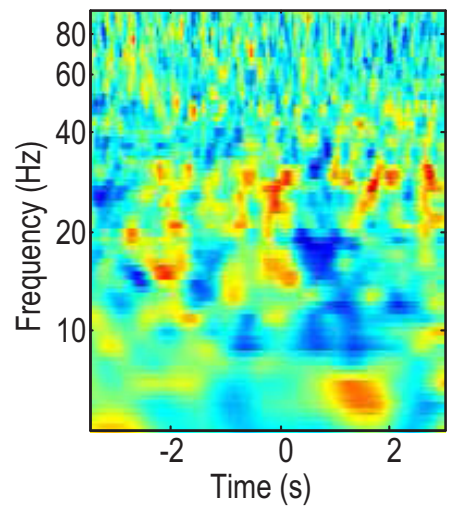

Index Finger: 10\% Effort

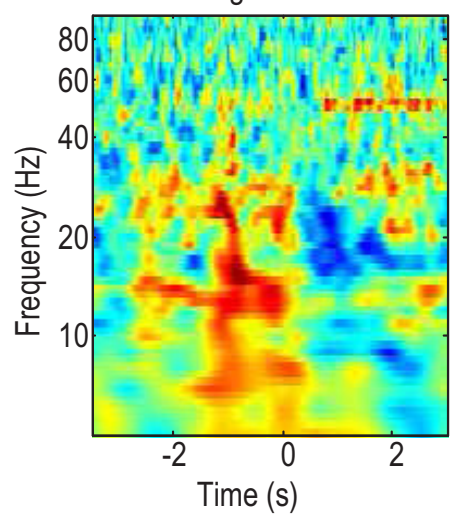

Little Finger: $25 \%$ Effort

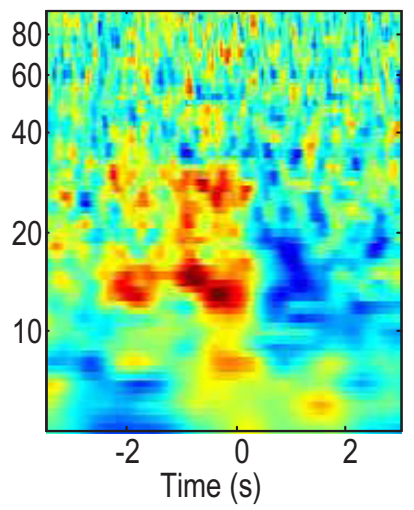

Index Finger: 25\% Effort

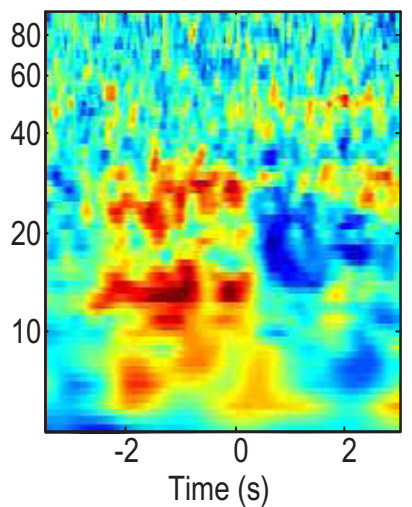

Little Finger: $40 \%$ Effort
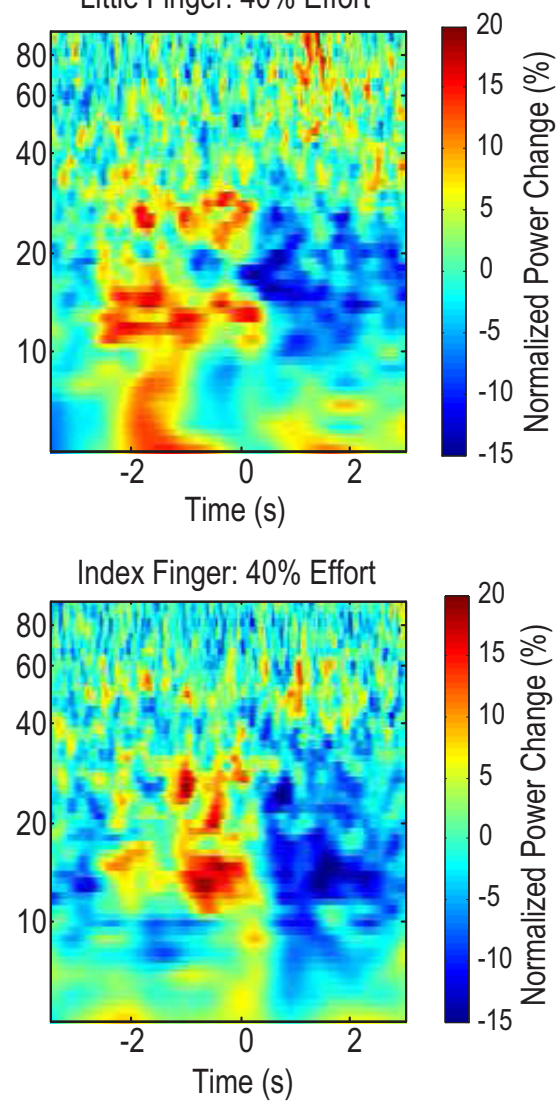

Figure 3. Average power changes aligned to the $\mathrm{Go}$ cue in the different experimental conditions. Power in the beta-band ( $13-30 \mathrm{~Hz}$ ) first increased after the warning cue (from minus 2.5 to $2.0 \mathrm{~s}$ ) and then reduced after the $\mathrm{Go}$ cue (time 0 in the figure). Both the increase and the decrease were significant with respect to baseline. Note line noise at $50 \mathrm{~Hz}$ in bottom panels.

and effort level (10\%, 25\%, and 40\% of the MVC of individual fingers) on the reaction time identified a significant effect of effort level $\left(F_{(2,30)}=12.728 ; p<0.001\right)$, but no effect of finger $\left(F_{(1,15)}=0.122\right.$; $p=0.732)$. The reaction time averaged across fingers reduced with increasing effort and was $556 \pm 31,477 \pm 37$, and $405 \pm 25 \mathrm{~ms}$ at effort levels of $10 \%, 25 \%$, and $40 \%$, respectively.
The force trajectory of each finger at different effort levels was normalized to the MVC force achieved by the index finger of the same hand and aligned to force onset for easier comparison across subjects (Fig. 2B). Similar results were seen if absolute force was also used (data not shown). Average normalized force during the second over which force was sta- 


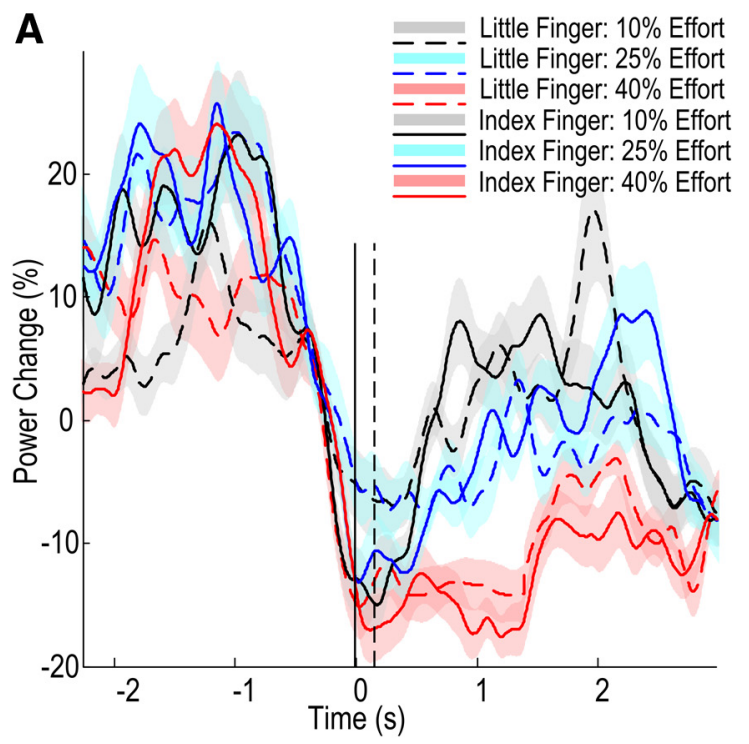

Figure 4. Beta activity during sustained contraction was modulated by effort level and not by the motor effector. $A$, Group mean \pm SEM (colored shaded areas) time evolving beta power aligned to force onset $(0 \mathrm{~s})$. The solid and dashed vertical lines indicate the time of force onset and the time to peak force rate, respectively. $\boldsymbol{B}$, Group mean \pm SEM beta power during the period of sustained contraction (taken from 1 to 2 s after the force onset; see Fig. 2).

ble (during sustained contraction in the middle of the gripping phase) was calculated for each condition. The "actual effort" measured as the average exerted force during the sustained contraction as a percentage of the MVC of the activated effector, closely matched the "required effort" set by the cue $(r=$ $0.978, p<0.001$ considering all individual trials from all subjects). Two-way ANOVA with the factors of finger (index or little) and effort level (10\%, 25\%, and 40\% of the MVC of individual fingers) applied on the exerted force identified significant effects of finger $\left(F_{(1,15)}=31.182, p<0.001\right)$ and effort $\left(F_{(2,30)}=789.28, p<0.001\right)$, together with a significant interaction between these two factors $\left(F_{(2,30)}=35.811, p<0.001\right)$. The force exerted by the index finger was significantly larger than that exerted by the little finger at each effort level: $9.02 \pm$ $0.35 \%$ vs $5.82 \pm 0.48 \%, t_{15}=4.808, p<0.001$ at effort level of $10 \% ; 23.43 \pm 0.31 \%$ vs $16.01 \pm 1.16 \%, t_{15}=5.446, p<0.001$ at effort level of $25 \%$; $38.11 \pm 0.27 \%$ vs $26.54 \pm 1.95 \%$, $t_{15}=5.827, p<0.001$ at effort level of $40 \%$. Therefore, effort and the actual exerted force were successfully dissociated in this paradigm.

The rate of the rise of force peaked shortly after force onset and then fell back to zero within one second of force onset, as shown in Figure $2 C$. The time interval between force onset and peak force rate $(108 \pm 8 \mathrm{~ms})$ was constant across all experimental conditions and a two-way ANOVA showed no effect of finger: $F_{(1,15)}=0.098 ; p=0.758$; no effect of effort: $F_{(2,30)}=$ $0.419 ; p=0.662$; nor an interaction: $F_{(2,30)}=2.659 ; p=0.086$. A two-way ANOVA of peak force rate with factors of finger and effort level identified a significant effect of finger $\left(F_{(1,15)}=\right.$ $40.745, p<0.001)$ and of effort $\left(F_{(2,30)}=58.006, p<0.001\right)$, but no interaction between these two factors $\left(F_{(2,30)}=2.857\right.$, $p=0.073)$. The maximal force rate achieved significantly correlated with the sustained stable force across subjects and across experimental conditions $(r=0.8230, p<0.001$, $n=64)$.
LFP beta activity increased after the warning cue and premovement beta ERPC positively correlated with reaction time

The average power changes during the preparation and movement relative to the average power before the warning cue are shown for different experimental conditions in Figure 3. The power in the betaband $(13-30 \mathrm{~Hz})$ increased to above baseline after the warning cue (average $\mathrm{ERPC}=5.45 \pm 1.99, t_{15}=2.739, p=$ 0.015 vs zero for the little finger across different efforts, average $\mathrm{ERPC}=8.89 \pm$ $1.56, t_{15}=3.476, p=0.003$ vs zero for the index finger, one-sample $t$ test) and then reduced to below baseline after the Go cue (average ERPC $=-10.42 \pm 1.72, t_{15}=$ -6.057, $p<0.001$ for the little finger across different efforts, average ERPC $=$ $-9.85 \pm 1.32, t_{15}=-1.52, p<0.001$ for the index finger, one-sample $t$ test).

Two-way ANOVA applied on the betaband ERPC during the preparation phase (between the warning cue and the Go cue) identified a significant effect of the finger $\left(F_{(1,15)}=5.696, p=0.031\right)$, but no effect of effort levels $\left(F_{(2,30)}=1.245 ; p=0.302\right)$ nor interaction between these factors $\left(F_{(1,15)}=0.502, p=0.610\right)$. The average beta ERPC was significantly larger when the index finger would be activated after the Go cue $(8.89 \pm 1.56$ ca $5.45 \pm 1.99$ when little finger would be activated, $\left.t_{15}=2.387, p=0.031\right)$. There was a positive correlation between the beta ERPC during the preparation phase and the reaction time after the Go cue across subjects and across experimental conditions $(r=0.4158, p<0.001, n=64)$.

We also calculated average beta power during the time between the Go cue and force onset and between force onset and the time to peak force rate, but there was no significant modulation effect of either effort or effector on the beta desynchronization during these periods of time and there was no consistent correlation between the beta desynchronization during these periods of time and the rate of the rise of muscle force.

LFP beta activity during sustained contraction was modulated by the effort level not by which finger was activated

Comparison of the event-related power change in different frequency bands for different experimental conditions revealed that LFP activity in the beta-band during a sustained contraction phase was suppressed as effort level increased (Fig. 4). Two-way ANOVA with the factors of finger (index or little) and effort level (10\%, 25\%, and $40 \%$ of MVC of that finger) was applied to the average beta relative power change during the second in the middle of the finger press when force was stable. This identified a significant effect of effort $\left(F_{(2,30)}=9.276, p=0.001, \eta^{2}=0.333\right)$ and a lack of effect of finger $\left(F_{(1,15)}=0.110, p=0.745, \eta^{2}=0.006\right)$ and no interaction between the finger and effort level $\left(F_{(2,30)}=1.774, p=0.187, \eta^{2}=\right.$ $0.106)$. In particular, the observed effect size of the effort level was 55.5 times that of the finger. Given the observed effect size for the finger, the statistical power for the finger would only increase by 0.002 even if the sample size were 10 times that of the current study. This indicates that the nonsignificant results for the effect of "finger" were not due to small sample size, but rather to the minute or negligible effect size. 
Table 2. AIC analysis results for different regression models for LFP beta activity during sustained contraction

\begin{tabular}{|c|c|c|c|c|c|c|}
\hline Model & Predictor & Effect of the predictor & AIC & $\Delta_{i}(A I C)$ & $w_{i}(A I C)$ & $\frac{w_{i}(A I C)}{w_{2}(A I C)}$ \\
\hline 1 & Required effort & $k=-0.278 \pm 0.043, p<0.001$ & 11453.9 & 3.2 & 0.168 & $p=0.202$ \\
\hline 3 & Absolute force & $k=-0.0159 \pm 0.007, p=0.021$ & 11472.2 & 21.5 & $1.78 \mathrm{e}-5$ & $p<0.001$ \\
\hline
\end{tabular}

Furthermore, multilevel linear modeling was applied to the data from all valid trials of all subjects, with the LFP beta activity during sustained contraction as the dependent variable, and the required effort (determined by the cue), actual effort (the average force during the sustained contraction phase as a percentage of the MVC of the specific effector), or the "absolute exerted force" as the predictor in different models. AIC analysis was used to evaluate different models (results in Table 2). This identified the model based on the actual effort as the best candidate model with the minimal AIC value, closely followed by the model with required effort as the predictor. Both models had significantly higher probability of being better, in terms of AIC, than the model using actual force as predictor. This is despite the observation that the absolute force by itself had a significant effect on the STN beta activity $(k=-0.0159 \pm 0.007, p=0.021)$. Modeling using the $z$-score of absolute force as the predictor showed that $1 \mathrm{SD}$ of increase in the absolute force led to a significant reduction $(4.266 \pm 0.574, p=0.020)$ in the STN beta ERS during movement. The significance of this effect was further confirmed by examining surrogate data. The model with absolute force had a significantly lower AIC value than the same model in which randomly permuted force was used as the predictor $(p=0.005$; $\mathrm{AIC}=11501.99 \pm 0.45$ for 1000 surrogates). Critically, though, the actual or required effort (the two being indistinguishable in the current paradigm) was a better predictor of LFP beta activity than the absolute exerted force in terms of AIC.

Consistent with this, considering the forces generated by different fingers together when the effort level was fixed, there was no significant correlation between the beta power change and force (normalized to index finger) achieved across subjects at any of the tested effort levels: $r=-0.0629, n=32, p=0.732$ at the effort level of 10\% of MVC of individual finger; $r=-0.2118, n=$ $32, p=0.245$ at the effort level of $25 \%$ of MVC; $r=-0.0944, n=$ $32, p=0.608$ at the effort level of $40 \%$ of MVC (Fig. 5). Similar results were seen if absolute force was used instead of force normalized to the index finger (data not shown).Therefore, our results indicated a significant effect of effort level with large effect size and statistical power, but little evidence for any effect of the finger used or the actual force generated in the modulation of the beta power during movement. The same analysis applied to relative gamma-band power during finger press failed to show any effect of effort $\left(F_{(2,30)}=1.197, p=0.316\right)$, or finger $\left(F_{(1,15)}=0.019, p=0.892\right)$ or their interaction $\left(F_{(2,30)}=\right.$ $2.230, p=0.125)$.

\section{Relationship between STN beta activity during sustained contraction and absolute force changed with motor effector} The dependency of the relationship between STN beta power suppression (-BetaERPC) during sustained contraction and absolute force upon motor effector is illustrated for an exemplar subject in Figure 6A. Multilevel linear modeling was applied to the data from all valid trials of all subjects to test different hypoth-

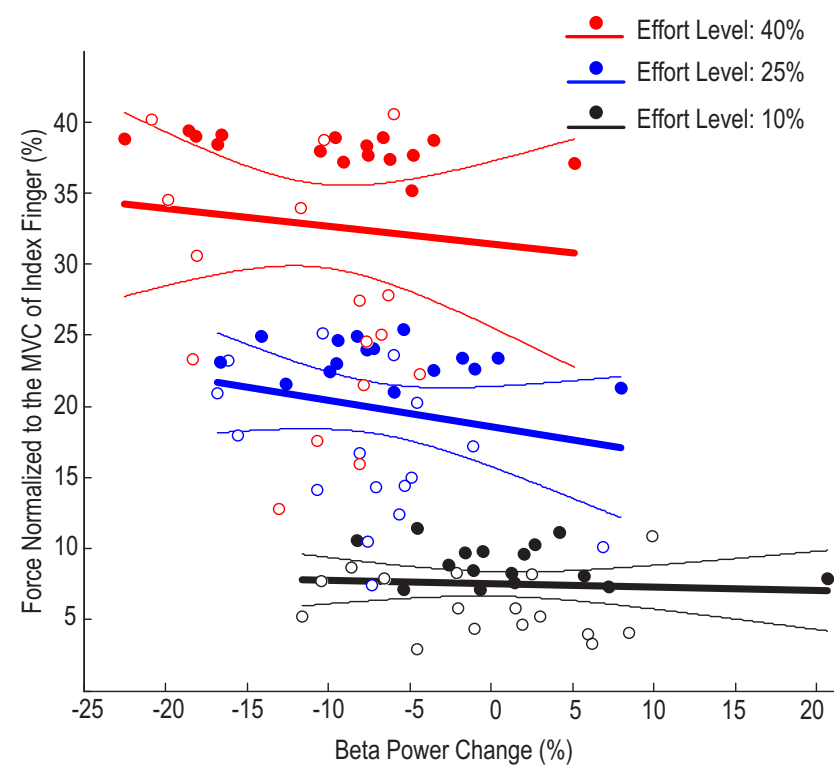

Figure 5. No significant correlation between normalized force and average beta power change during movement at each effort level when forces from different fingers were considered together. Regression lines and their respective $95 \%$ confidence limits are shown. Filled circles are data points from the index fingers; unfilled circles are data from little fingers.

eses about the relationship of STN beta activity, motor effector, and the absolute exerted force. Four models were compared. The first (Equation 1) assumed that the absolute exerted force scaled with beta power suppression (-BetaERPC) with a constant regression coefficient and that any effect exerted by the different effectors consisted of an intercept shift. The second model (Equation 2) differed from the first model in the inclusion of an additional term of interaction between the relative beta suppression and the motor effector. This term modeled the effects of different motor effectors as changing the regression coefficient, with this change constant across all subjects. In the third model (Equation 3 ), the absolute force scaled with the beta power suppression and the regression coefficient scaled with the MVC of the motor effector. The intercept shift introduced by different effectors remained. The fourth model (Equation 4) tested whether an extra interaction term between the relative beta suppression and motor effector could further improve the prediction of exerted force if the slope between the force and the beta change was modeled as scaling with the MVC of the motor effector as in Model 3.

$$
\text { AbsForce }=k_{1} \cdot(- \text { BetaERPC })+k_{2} \cdot \text { Finger }+k_{3}
$$

where Finger $=0$ for little finger, and 1 for index finger

AbsForce $=k_{1} \cdot(-$ BetaERPC $)+k_{2} \cdot$ 
A

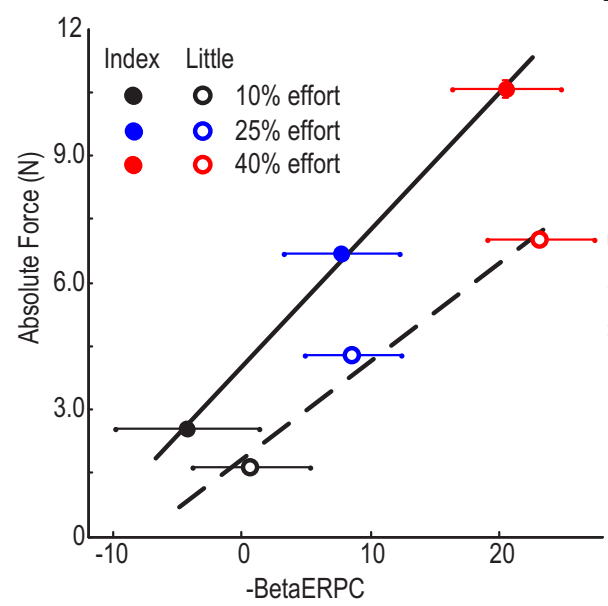

B

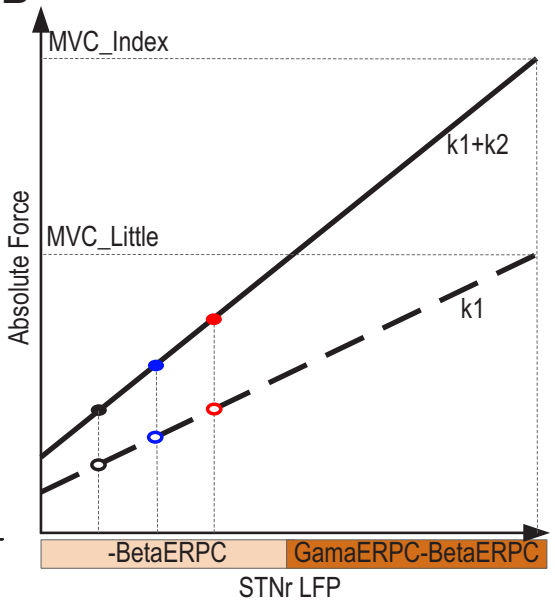

Figure 6. Dependency of force generation on finger in an exemplar subject $(\boldsymbol{A})$ and in a schematic of modeling $(\boldsymbol{B})$. $\boldsymbol{A}$, Data from case 7 (right hand). The solid and dashed line show the regression lines for the absolute force of the index and little fingers, respectively. The dots with horizontal bars show the means and SEMs across trials for different conditions. Note that in $\boldsymbol{A}$ and $\boldsymbol{B}$, the sign of the event-related LFP power change is reversed (-BetaERPC) for convenience of viewing; therefore, more positive values represent increasing beta suppression. $\boldsymbol{B}$, Actual exerted force scales with STN LFP beta power suppression relative to baseline and the maximal capacity of the effector adopted in the task. Thick solid line and dashed line in the figure show the force generation from effectors of different MVCs. The basal ganglia help determine the effort or vigor to be attributed to a response, with beta suppression operationalizing, or providing a proxy for, effort during sustained finger pressing. Note that gamma-band power increases in the STN LFP relative to baseline (GammaERPC) may be enlisted in coding effort at stronger effort levels (as suggested by Tan et al., 2013b). When different effectors are adopted, beta modulation is similar at the same effort level, but the actual force generated is different (compare colored filled circles with unfilled circles).

$$
\begin{aligned}
&(- \text { BetaERPC }) \cdot \text { Finger }+k_{3} \cdot \text { Finger }+k_{4} \\
& \text { AbsForce }= k_{1} \cdot M C_{i} \cdot(- \text { BetaERPC })+k_{2} \cdot \text { Finger }+k_{3}
\end{aligned}
$$

where $M V C_{i}$ the MVC of the motor effector

(little finger or index finger)

$$
\begin{aligned}
\text { AbsForce }= & k_{1} \cdot M_{V C_{i}} \cdot(- \text { BetaERPC })+k_{2} \cdot \\
& (- \text { BetaERPC }) \cdot \text { Finger }+k_{3} \cdot \text { Finger }+k_{4}
\end{aligned}
$$

Model 2 identified a significant effect of relative beta LFP power $\left(k_{1}=0.027 \pm 0.0065, p<0.001\right)$ and a significant interaction between the beta power and motor effector $\left(k_{2}=0.033 \pm 0.010\right.$, $p=0.0017)$ in predicting the absolute force generated. This suggests that the regression coefficients relating relative beta LFP power suppression to the absolute force generated were significantly different for the two fingers, with the regression coefficient having larger absolute values for the index finger. Model 3 identified a significant effect of the $M V C_{i} \cdot(-$ BetaERPC $)\left(k_{1}=0.013 \pm 0.001, p<\right.$ 0.001 ), confirming that the regression coefficients between beta power suppression and the absolute force $k_{1} \cdot M V C_{i}$ scaled with the MVC of the motor effector. Model 4 showed that the interaction between the motor effector and the beta suppression was no longer necessary for the prediction of exerted force $\left(k_{2}=\right.$ $0.0056 \pm 0.0037, p=0.418$ ) once the regression coefficients between the beta power suppression and force was modeled as scaling with the MVC of the effector. The AIC analysis (results shown in Table 3) identified Model 3 to be the more compact and accurate representation of the relationship between the beta ERPC and exerted force and to have significantly higher probability of being the better model than the other two in terms of AIC. Therefore, absolute force scaled with beta power suppression but with

the scaling determined by the MVC of the motor effector, which is consistent with our hypothesis that it is effort (i.e., force as a fraction of the total force achievable by the corresponding effector) that is indexed by beta LFP power suppression.

\section{Discussion}

The basal ganglia motor cortical circuit, including its dopaminergic innervation, has been suggested to regulate response vigor or effort (Niv and Rivlin-Etzion, 2007; Salamone et al., 2009; Turner and Desmurget, 2010). Previous studies have shown that frequency-specific LFP activities in the STN and globus pallidus correlate with effort and force in manual actions over the full range of contraction strengths in patients with PD or dystonia (Brücke et al., 2012; Tan et al., 2013b). Using motor effectors of different capacities for force generation, we dissociated effort from the actual force of the motor response. In particular, we show that beta power changes in the STN LFP negatively correlate with effort level independently of the motor effector or the actual force that was exerted when the effort level was stable. Our results support the hypothesis that the basal ganglia help determine the effort or vigor to be attributed to a response rather than being directly involved in the parameterization of the motor response in terms of absolute force. The results suggest that the beta suppression in the STN LFP during sustained contraction serves as or provides a proxy for effort (albeit at low effort levels) and that actual force scales with the effort signal from the basal ganglia and the maximal capacity of the effector adopted in the task in the manner schematically illustrated in Figure 6B. At stronger efforts, STN gamma power increases are enlisted in coding effort (Anzak et al., 2012; Tan et al., 2013b).

Before considering the significance of the findings in greater detail, we should acknowledge some significant limitations of the present study. First, participants were necessarily PD patients who had undergone surgery, so inferences with regard to normal functioning must be circumspect (Williams et al., 2002). That said, recordings were made on levodopa while patients' behavioral state was as near normal as possible, albeit not normal. Second, we limited maximal effort level to $40 \%$ of the MVC of individual fingers to limit fatigue. We have previously shown that force or effort correlate with beta band desynchronization over this range (Tan et al., 2013b). Higher forces or efforts were not tested and it is under these conditions that gamma synchronization may play a role in encoding force or effort. Therefore, the current study was focused on lower effort levels where beta desynchronization preferentially scales with effort, but was not designed to explore gamma power changes. Third, because visual feedback of exerted effort and required effort were simultaneously presented and veridical, we cannot dissociate between these two aspects of effort and any distinction remains an interesting research question for further study. However, we could show that the beta synchronization was not modulated by effort during the preparation phase between the warning cue and the go cue, providing some evidence that the correlation between beta activity and effort was related to motor performance rather than elicited 
Table 3. AIC analysis results for different models describing how absolute force relates to LFP beta activity during sustained contraction

\begin{tabular}{|c|c|c|c|c|c|c|c|}
\hline Model & Predictor & Effects & $d f$ & AIC & $\Delta_{i}(A I C)$ & $w_{i}(A I C)$ & $\frac{w_{i}(A I C)}{w_{3}(A I C)}$ \\
\hline 1 & Beta + finger & $\begin{array}{l}\text { Beta: } k=0.039 \pm 0.005, p<0.001 ; \text { finger: } k= \\
\quad 2.626 \pm 0.203, p<0.001\end{array}$ & 5 & 7183.41 & 29.76 & 2.081e-7 & $p<0.001$ \\
\hline 2 & Beta + finger + beta*finger & $\begin{array}{l}\text { Beta: } k=0.027 \pm 0.006, p<0.001 ; \text { finger: } k= \\
2.593 \pm 0.203, p<0.001 ; \text { beta }{ }^{*} \text { finger: } k= \\
0.033 \pm 0.010, p=0.0017\end{array}$ & 6 & 7175.52 & 21.87 & $1.076 \mathrm{e}-5$ & $p<0.001$ \\
\hline 4 & Beta*MVC + finger + beta*finger & $\begin{array}{l}\text { Beta*MVC: } k=0.013 \pm 0.001, p<0.001 ; \text { finger: } \\
\quad k=2.609 \pm 0.202, p<0.001 ; \\
\quad \text { beta* }^{*} \text { finger: } k=0.0056 \pm 0.0037, p=0.418\end{array}$ & 6 & 7154.49 & 0.84 & 0.396 & 0.656 \\
\hline
\end{tabular}

Model 3 was the model with the minimal AIC value and the extra interaction term in Model 4 did not further increase the prediction power of the model. The Akaike weight $\left(w_{i}(A I C)\right)$ and the relative $A$ kaike weight $\frac{w_{i}(A I C)}{w_{3}(A I C)}$ showed that Model 3 had the highest probability of being the best model.

by signaling of the required effort level. Fourth, the multilevel linear modeling combined with model comparison and selection approach based on AIC used in part here, as with any other model-based approach, can select the most compact and accurate description of the relationship of interest, but only from among presented candidate models. Finally, by only recording from the locality of the STN we can only assert that this nucleus may help to determine the effort or vigor to be attributed to a motor response because this function may be underpinned by a more extensive circuit that includes, but is not limited to, the STN.

The current study provides evidence for a contribution of the basal ganglia to encoding effort rather than actual force. Interestingly, this function was manifest during sustained contraction and not before even though subjects were presented with predictive information about the task. Indeed, once this predictive information was presented beta activity rose, rather as seen in no-go tasks (Kühn et al., 2004), and perhaps indicating the need to suppress intercurrent processing in readiness for the imperative cue. This change in beta activity during a period without movement underscores the fact that changes in beta synchrony do not themselves exclusively code for any single parameter or function. Rather, they may be better conceptualized as a property of basal ganglia circuits that sets the performance of circuit elements (Brittain and Brown, 2014). Beta synchronization may also be modulated when independent or overlapping circuits are engaged in other functions, such as offline error correction mechanisms (Tan et al., 2014) or the suppression of conflicting choices affecting behavior (Brittain et al., 2012). In this schema, beta synchrony is a generic feature of basal ganglia circuits, which is then modulated according to the recruitment of specific network elements that are necessary in the delivery of the task at any given moment in time. Such a schema therefore also allows for changes in beta activity to be a composite of several simultaneously active lines of processing.

The notion that one of the functions of the basal ganglia is to encode effort rather than force is consistent with findings from previous studies in which subjects were required to match different levels of absolute force or torque between two limbs with asymmetric maximum strength whether this was induced by fatigue (Carson et al., 2002; Weerakkody et al., 2003; Proske et al., 2004) or naturally present (Simon and Ferries, 2008). These studies showed that, without visual feedback, subjects attempting to produce equal forces between limbs actually produce equal relative forces (i.e., scaled to the instantaneous maximum strength of the muscle groups), consistent with an interpretation based on a matching of effort rather than absolute force or torque and consistent with our hypothesis about the central encoding of effort as opposed to absolute force. In addition, the current results have two interesting implications. First, our results suggested that the basal ganglia help to determine the effort to be attributed to a motor response, with such effort being related to the current maximal capacity of the motor effector. The relationship between motor effort and absolute force may change when tasks are executed with different effectors, such as the index and little finger as tested here, and due to fatigue, illness, or trauma. Therefore, to generate the most appropriate motor response required for a certain task, the required effort and underpinning frequencyspecific basal ganglia activities need to be adjusted and to take into account the current state of available effectors. This adjustment may depend on peripheral feedback revealing unexpected changes in the properties of motor effectors and/or on an internal forward model that includes representation of the dynamic range of forces generated by different effectors given a certain effort level. Such an internal forward model, which generates predictions of the state of the system given current motor commands and permits compensation for instabilities due to feedback delays and uncertainty, is assumed to be a central feature in motor control, particularly during ballistic movements (Wolpert and Miall, 1996; Todorov and Jordan, 2002; Diedrichsen et al., 2010). This implies that the function of the basal ganglia in optimal control might not be limited to learning costs and rewards (Shadmehr and Krakauer, 2008), but might also extend to updating the required motor effort based on predictions from internal forward models. Second, the present findings may provide insight into the pathophysiology of bradykinesia in PD. Previous work has shown that, off of dopaminergic medication, the power suppression in the beta-band, especially that in the low-beta-band $(13-23 \mathrm{~Hz})$, was attenuated in patients with PD during movement initialization (Doyle et al., 2005; Devos et al., 2006; Androulidakis et al., 2007; Anzak et al., 2012) and when a constant force was meant to be sustained (Tan et al., 2013a). Such a diminished range of betaband suppression during movement may restrict the dynamic range of the coding of effort (contracting the $x$-axis in Fig. $6 B$ ) and contribute to underscaled, bradykinetic movements. This is consistent with previous observations that untreated PD patients produce normal muscle activation patterns, but that muscle activity is not adequately scaled to produce the required force (Berardelli et al., 1986; Turner and Desmurget, 2010), and that PD patients show an increased probability of selecting slow movement speeds (Mazzoni et al., 2007). The relative reversibility of these motor deficits and improvement in motor-related beta reactivity with dopaminergic therapy further implicates the dopaminergic system in transcribing behavioral expediency into 
motor effort (Schmidt et al., 2008), a process that seems to involve, or be indexed by, beta-band suppression.

\section{References}

Androulidakis AG, Kühn AA, Chen CC, Blomstedt P, Kempf F, Küpsch A, Schneider GH, Doyle L, Dowsey-Limousin P, Hariz MI, Brown P (2007) Dopaminergic therapy promotes lateralized motor activity in the subthalamic area in Parkinson's disease. Brain 130:457-468. CrossRef Medline

Anzak A, Tan H, Pogosyan A, Foltynie T, Limousin P, Zrinzo L, Hariz M, Ashkan K, Bogdanovic M, Green AL, Aziz T, Brown P (2012) Subthalamic nucleus activity optimizes maximal effort motor responses in Parkinson's disease. Brain 135:2766-2778. CrossRef Medline

Baraduc P, Thobois S, Gan J, Broussolle E, Desmurget M (2013) A common optimization principle for motor execution in healthy subjects and parkinsonian patients. J Neurosci 33:665-677. CrossRef Medline

Berardelli A, Dick JP, Rothwell JC, Day BL, Marsden CD (1986) Scaling of the size of the first agonist EMG burst during rapid wrist movements in patients with Parkinson's disease. J Neurol Neurosurg Psychiatry 49: 1273-1279. CrossRef Medline

Brittain JS, Brown P (2014) Oscillations and the basal ganglia: motor control and beyond. Neuroimage 85:637-647. CrossRef Medline

Brittain JS, Watkins KE, Joundi RA, Ray NJ, Holland P, Green AL, Aziz TZ, Jenkinson N (2012) A role for the subthalamic nucleus in response inhibition during conflict. J Neurosci 32:13396-13401. CrossRef Medline

Brücke C, Huebl J, Schönecker T, Neumann WJ, Yarrow K, Küpsch A, Blahak C, Lütjens G, Brown P, Krauss JK, Schneider GH, Kühn AA (2012) Scaling of movement is related to pallidal gamma oscillations in patients with dystonia. J Neurosci 32:1008-1019. CrossRef Medline

Burnham KP, Anderson DR (2002) Model selection and multimodel inference: a practical information-theoretic approach. New York: Springer.

Carson RG, Riek S, Shahbazpour N (2002) Central and peripheral mediation of human force sensation following eccentric or concentric contractions. J Physiol 539:913-925. CrossRef Medline

DeLong MR, Alexander GE, Georgopoulos AP, Crutcher MD, Mitchell SJ, Richardson RT (1984) Role of basal ganglia in limb movements. Hum Neurobiol 2:235-244. Medline

Devos D, Szurhaj W, Reyns N, Labyt E, Houdayer E, Bourriez JL, Cassim F, Krystkowiak P, Blond S, Destée A, Derambure P, Defebvre L (2006) Predominance of the contralateral movement-related activity in the subthalamo-cortical loop. Clin Neurophysiol 117:2315-2327. CrossRef Medline

Diedrichsen J, Shadmehr R, Ivry RB (2010) The coordination of movement: optimal feedback control and beyond. Trends Cogn Sci 14:31-39. CrossRef Medline

Doyle LM, Kühn AA, Hariz M, Küpsch A, Schneider GH, Brown P (2005) Levodopa-induced modulation of subthalamic beta oscillations during self-paced movements in patients with Parkinson's disease. Eur J Neurosci 21:1403-1412. CrossRef Medline

Erdfelder E, Faul F, Buchner A (1996) GPOWER: a general power analysis program. Behav Res Methods Instr Comput 28:1-11.

Faul F, Erdfelder E, Lang AG, Buchner A (2007) G*Power 3: a flexible statistical power analysis program for the social, behavioral, and biomedical sciences. Behav Res Methods 39:175-191. Medline

Foltynie T, Hariz MI (2010) Surgical management of Parkinson's disease. Expert Rev Neurother 10:903-914. CrossRef Medline

Guigon E, Baraduc P, Desmurget M (2007) Computational motor control: redundancy and invariance. J Neurophysiol 97:331-347. CrossRef Medline

Hox JJ (2002) Multilevel analysis: techniques and applications. Mahwah, NJ: Lawrence Erlbaum Associates.

Joundi RA, Brittain JS, Green AL, Aziz TZ, Brown P, Jenkinson N (2012)
Oscillatory activity in the subthalamic nucleus during arm reaching in Parkinson's disease. Exp Neurol 236:319-326. CrossRef Medline

Kühn AA, Williams D, Küpsch A, Limousin P, Hariz M, Schneider GH, Yarrow K, Brown P (2004) Event-related beta desynchronization in human subthalamic nucleus correlates with motor performance. Brain 127: 735-746. CrossRef Medline

Mazzoni P, Hristova A, Krakauer JW (2007) Why don't we move faster? Parkinson's disease, movement vigor, and implicit motivation. J Neurosci 27:7105-7116. CrossRef Medline

Niv Y, Rivlin-Etzion M (2007) Parkinson's disease: fighting the will? J Neurosci 27:11777-11779. CrossRef Medline

Pinheiro J, Bates D, DebRoy S, Sarkar D, R Development Core Team (2013) Nlme: linear and nonlinear mixed effects models. R package version 3.1-113.

Prodoehl J, Corcos DM, Vaillancourt DE (2009) Basal ganglia mechanisms underlying precision grip force control. Neurosci Biobehav Rev 33:900 908. CrossRef Medline

Proske U, Gregory JE, Morgan DL, Percival P, Weerakkody NS, Canny BJ (2004) Force matching errors following eccentric exercise. Hum Mov Sci 23(3-4):365-378.

Salamone JD, Correa M, Farrar AM, Nunes EJ, Pardo M (2009) Dopamine, behavioral economics, and effort. Front Behav Neurosci 3:13. Medline

Schmidt L, d'Arc BF, Lafargue G, Galanaud D, Czernecki V, Grabli D, Schüpbach M, Hartmann A, Lévy R, Dubois B, Pessiglione M (2008) Disconnecting force from money: effects of basal ganglia damage on incentive motivation. Brain 131:1303-1310. Medline

Shadmehr R, Krakauer JW (2008) A computational neuroanatomy for motor control. Exp Brain Res 185:359-381. CrossRef Medline

Simon AM, Ferris DP (2008) Lower limb force production and bilateral force asymmetries are based on sense of effort. Exp Brain Res 187:129_ 138. CrossRef Medline

Spraker MB, Yu H, Corcos DM, Vaillancourt DE (2007) Role of individual basal ganglia nuclei in force amplitude generation. J Neurophysiol 98: 821-834. CrossRef Medline

Tan H, Pogosyan A, Anzak A, Foltynie T, Limousin P, Zrinzo L, Ashkan K, Bogdanovic M, Green AL, Aziz T, Brown P (2013a) Frequency specific activity in subthalamic nucleus correlates with hand bradykinesia in Parkinson's disease. Exp Neurol 240:122-129. CrossRef Medline

Tan H, Pogosyan A, Anzak A, Ashkan K, Bogdanovic M, Green AL, Aziz T, Foltynie T, Limousin P, Zrinzo L, Brown P (2013b) Complementary roles of different oscillatory activities in the subthalamic nucleus in coding motor effort in Parkinsonism. Exp Neurol 248:187-195. CrossRef Medline

Tan H, Jenkinson N, Brown P (2014) Dynamic neural correlates of motor error monitoring and adaptation during trial-to-trial learning. J Neurosci 34:5678-5688. CrossRef Medline

Todorov E, Jordan MI (2002) Optimal feedback control as a theory of motor coordination. Nat Neurosci 5:1226-1235. CrossRef Medline

Turner RS, Anderson ME (1997) Pallidal discharge related to the kinematics of reaching movements in two dimensions. J Neurophysiol 77:1051-1074. Medline

Turner RS, Desmurget M (2010) Basal ganglia contributions to motor control: a vigorous tutor. Curr Opin Neurobiol 20:704-716. CrossRef Medline

Wagenmakers EJ, Farrell S (2004) AIC model selection using Akaike weights. Psychon Bull Rev 11:192-196. CrossRef Medline

Weerakkody N, Percival P, Morgan DL, Gregory JE, Proske U (2003) Matching different levels of isometric torque in elbow flexor muscles after eccentric exercise. Exp Brain Res 149:141-150. Medline

Williams D, Tijssen M, Van Bruggen G, Bosch A, Insola A, Di Lazzaro V, Mazzone P, Oliviero A, Quartarone A, Speelman H, Brown P (2002) Dopaminedependent changes in the functional connectivity between basal ganglia and cerebral cortex in humans. Brain 125:1558-1569. CrossRef Medline

Wolpert DM, Miall RC (1996) Forward Models for Physiological Motor Control. Neural Netw 9:1265-1279. CrossRef Medline 